\title{
Generalized Z-contraction on quasi metric spaces and a fixed point result
}

\author{
Hakan Şimşek*, Menşur Tuğba Yalçın \\ Department of Mathematics, Faculty of Science and Arts, Kirikkale University, 71450 Yahsihan, Kirikkale, Turkey. \\ Communicated by B. Samet
}

\begin{abstract}
The simulation function is defined by Khojasteh et al. [F. Khojasteh, S. Shukla, S. Radenović, Filomat, 29 (2015), 1189-1194]. Khojasteh introduced the notion of Z-contraction which is a new type of nonlinear contractions defined by using a specific simulation function. Then, they proved existence and uniqueness of fixed points for Z-contraction mappings. After this work, studies involving simulation functions were performed by various authors [H. H. Alsulami, E. Karapinar, F. Khojasteh, A. F. Roldán-López-de-Hierro, Discrete Dyn. Nat. Soc., 2014 (2014), 10 pages], [M. Olgun, Ö. Biçer, T. Alyildiz, Turkish J. Math., 40 (2016), 832-837]. In this paper, we introduce generalized simulation function on a quasi metric space and we present a fixed point theorem. (C)2017 All rights reserved.
\end{abstract}

Keywords: Quasi metric space, left K-Cauchy sequence, simulation functions, fixed point.

2010 MSC: $47 \mathrm{H} 10,54 \mathrm{H} 25$.

\section{Introduction and preliminaries}

The theory of metric spaces takes an important place in the transition from classical analysis to modern analysis. Many concepts of topology are transmitted from metric spaces. One of the most remarkable works on functions defined in metric spaces was done by Banach [3]. Various generalizations of Banach fixed point theorem were made by numerous mathematicians $[4-7,9,10,12,13,15,18$, 20]. There exist many different definitions obtained by removing the symmetry axiom in metric spaces. The "quasimetric" concept first introduced by Wilson [21] in 1931. There have been several studies on this area since then $[8,11,16,17,19]$.

Definition 1.1. Let $X$ be any set. Then a function $d: X \times X \rightarrow[0, \infty)$ is said to be a quasi metric on $X$ if it has the following properties for all $x, y, z \in X$;

1) $\mathrm{d}(x, x)=0$;

2) $d(x, y) \leqslant d(x, z)+d(z, y)$;

3) $d(x, y)=d(y, x)=0 \Rightarrow x=y$.

\footnotetext{
${ }^{*}$ Corresponding author

Email addresses: hsimsek@kku.edu.tr:hasimsek@hotmail.com (Hakan Şimşek), tuugbaa@hotmail. com (Menşur Tuğba Yalçın)
}

doi:10.22436/jnsa.010.07.03 
If a quasi metric $d$ satisfies the additional condition

4) $d(x, y)=0 \Rightarrow x=y$,

then $d$ is said to be $T_{1}$-quasi metric.

Remark 1.2. It is clear that, every metric is a $\mathrm{T}_{1}$-quasi metric, every $\mathrm{T}_{1}$-quasi metric is a quasi metric.

Definition 1.3. A quasi (resp. $T_{1}$-quasi) metric space is a pair $(X, d)$ such that $X$ is a nonempty set and $\mathrm{d}$ is a quasi (resp. $T_{1}$-quasi) metric on $X$. Given a quasi metric space $(X, d)$ and any real number $\varepsilon>0$, the open ball and closed ball, respectively, of radius $\varepsilon$ and center $x_{0} \in X$ are the sets $B_{d}\left(x_{0}, \varepsilon\right) \subset X$ and $\mathrm{B}_{\mathrm{d}}\left[\mathrm{x}_{0}, \varepsilon\right] \subset \mathrm{X}$ defined by

$$
B_{d}\left(x_{0}, \varepsilon\right)=\left\{y \in X: d\left(x_{0}, y\right)<\varepsilon\right\},
$$

and

$$
\mathrm{B}_{\mathrm{d}}\left[\mathrm{x}_{0}, \varepsilon\right]=\left\{\mathrm{y} \in \mathrm{X}: \mathrm{d}\left(\mathrm{x}_{0}, \mathrm{y}\right) \leqslant \varepsilon\right\} .
$$

Every quasi metric $d$ on $X$ generates a natural topology on $X$. This topology on $X$ generated by the family of open balls $\left\{B_{d}(x, \varepsilon): x \in X\right.$ and $\left.\varepsilon>0\right\}$ as base is called the topology $\tau_{d}$ (or, the topology induced by the quasi metric $d)$.

Remark 1.4. If $(X, d)$ is a quasi metric space, then $\tau_{d}$ is a $T_{0}$ topology, and if $(X, d)$ is a $T_{1}$-quasi metric space, then $\tau_{d}$ is a $T_{1}$ topology.

Definition 1.5. Let $\left\{x_{n}\right\}$ be a sequence in the quasi metric space $(X, d)$ and $x \in X$. The sequence $\left\{x_{n}\right\}$ is said to be convergent to $x$ with respect to $\tau_{d}$ if and only if

$$
\lim _{n \rightarrow \infty} d\left(x_{n}, x\right)=\lim _{n \rightarrow \infty} d\left(x, x_{n}\right)=0 .
$$

There are many different approaches to Cauchy sequences in quasi-metric spaces. Reilly et al. [16] defined six different Cauchy sequences, three of them are as follows:

Definition 1.6. A sequence $\left\{x_{n}\right\}$ in a quasi metric space $(X, d)$ is called

- left K-Cauchy if for every $\varepsilon>0$, there exists $n_{0} \in \mathbb{N}$ such that

$$
\forall n, k, n \geqslant k \geqslant n_{0}, d\left(x_{k}, x_{n}\right)<\varepsilon ;
$$

- right $\mathrm{K}$-Cauchy if for every $\varepsilon>0$, there exists $\mathrm{n}_{0} \in \mathbb{N}$ such that

$$
\forall n, k, n \geqslant k \geqslant n_{0}, d\left(x_{n}, x_{k}\right)<\varepsilon ;
$$

- d-Cauchy if for every $\varepsilon>0$, there exists $n_{0} \in \mathbb{N}$ such that

$$
\forall n, k \geqslant n_{0}, d\left(x_{n}, x_{k}\right)<\varepsilon .
$$

Remark 1.7. It is obvious that a sequence is d-Cauchy if and only if it is both left K-Cauchy and right K-Cauchy.

In order for a metric space to be complete, the necessary and sufficient condition is that, every Cauchy sequence in this space is convergent. However, in a quasi-metric space, completeness cannot be uniquely defined. Altun et al. classified nine different definitions of completeness [2], three of them are the following:

Definition 1.8. A quasi metric space $(X, d)$ is called 
- left $\mathcal{K}$-complete if every left $\mathrm{K}$-Cauchy sequence is convergent;

- right $\mathcal{K}$-complete if every right $\mathrm{K}$-Cauchy sequence is convergent;

- $\zeta$-complete if every every d-Cauchy sequence is convergent.

In this paper we will use d-Cauchyness and $\zeta$-completeness by simply saying Cauchyness and completeness respectively.

The simulation function is defined by Khojasteh et al. in 2015 [14].

Definition 1.9. A simulation function is a mapping $\zeta:[0, \infty) \times[0, \infty) \rightarrow \mathbb{R}$ satisfying the following conditions:

$(\zeta 1) \zeta(0,0)=0$;

(द2) $\zeta(t, s)<s-t, \forall t, s>0$;

(¿3) If $\left\{t_{n}\right\}$ and $\left\{s_{n}\right\}$ are two sequences in $(0, \infty)$ such that $\lim _{n \rightarrow \infty} t_{n}=\lim _{n \rightarrow \infty} s_{n}>0$, then $\limsup _{n \rightarrow \infty} \zeta\left(t_{n}, s_{n}\right)<0$.

Let $Z$ be the family of all simulation functions $\zeta:[0, \infty) \times[0, \infty) \rightarrow \mathbb{R}$.

Definition 1.10 ([4]). A self-mapping $T: X \rightarrow X$ on quasi metric space $(X, d)$ is called

- asymptotically right regular at a point $x \in X$ if

$$
\lim _{n \rightarrow \infty} d\left(T^{n-1} x, T^{n} x\right)=0 ;
$$

- asymptotically left regular at a point $x \in X$ if

$$
\lim _{n \rightarrow \infty} d\left(T^{n} x, T^{n-1} \chi\right)=0 ;
$$

- asymptotically regular if it is both asymptotically left regular and right regular.

It is known that every $Z$ contraction on a quasi metric space is asymptotically regular [1].

The aim of this paper is to present a new fixed point result for simulation function contractive mappings on complete quasi metric spaces.

\section{Fixed point results}

First let us define generalized $\mathrm{Z}$ contraction inspired by Rhoades type generalizations [17].

Definition 2.1. Let $(X, d)$ be a quasi metric space and $T: X \rightarrow X$ be a mapping. Then $T$ is said to be a generalized $Z$ contraction if it satisfies

$$
\zeta(d(T x, T y), M(x, y)) \geqslant 0,
$$

for all $x, y \in X$, where $\zeta \in Z$ and

$$
M(x, y)=\max \left\{d(x, y), d(T x, x), d(T y, y), \frac{1}{2}[d(x, T y)+d(T x, y)]\right\} .
$$

Lemma 2.2. Every generalized $Z$ contraction mapping on a quasi metric space is asymptotically regular.

Proof. Let $(X, d)$ be a quasi metric space and let $T: X \rightarrow X$ be a generalized $Z$ contraction with respect to $\zeta \in Z$. If $T^{p} \chi=T^{p-1} \chi$ for some $p \in \mathbb{N}$, then

$$
y=T^{p-1} x
$$

is a fixed point of $\mathrm{T}$. Therefore we have 


$$
\begin{aligned}
d\left(T^{n} x, T^{n+1} \chi\right) & =d\left(T^{n-p+1} T^{p-1} \chi, T^{n-p+2} T^{p-1} \chi\right)=d\left(T^{n-p+1} y, T^{n-p+2} y\right) \\
& =d(y, y)=0
\end{aligned}
$$

Thus $\lim _{n \rightarrow \infty} d\left(T^{n} \chi, T^{n+1} \chi\right)=0$ holds. So let $T^{n} \chi \neq T^{n-1} \chi$, for all $n \in \mathbb{N}$. It is known that

$$
\zeta\left(d\left(T^{n+1} x, T^{n} x\right), M\left(T^{n} x, T^{n-1} x\right)\right) \geqslant 0,
$$

since $T$ is a generalized $Z$ contraction, where

$$
\begin{gathered}
M\left(T^{n} x, T^{n-1} x\right)=\max \left\{d\left(T^{n} x, T^{n-1} x\right), d\left(T^{n+1} \chi, T^{n} x\right), d\left(T^{n} x, T^{n-1} \chi\right),\right. \\
\left.\quad \frac{1}{2}\left(d\left(T^{n} x, T^{n} x\right)+d\left(T^{n+1} x, T^{n-1} \chi\right)\right)\right\} \\
=\max \left\{d\left(T^{n} x, T^{n-1} x\right), d\left(T^{n+1} x, T^{n} x\right)\right\},
\end{gathered}
$$

since

$$
d\left(T^{n+1} x, T^{n-1} x\right) \leqslant d\left(T^{n+1} x, T^{n} x\right)+d\left(T^{n} x, T^{n-1} \chi\right) .
$$

If $\max \left\{d\left(T^{n} \chi, T^{n-1} \chi\right), d\left(T^{n+1} \chi, T^{n} x\right)\right\}=d\left(T^{n+1} \chi, T^{n} \chi\right)$, then

$$
\zeta\left(d\left(T^{n+1} \chi, T^{n} x\right), M\left(T^{n} \chi, T^{n-1} x\right)\right)=\zeta\left(d\left(T^{n+1} \chi, T^{n} x\right), d\left(T^{n+1} x, T^{n} x\right)\right) \geqslant 0,
$$

contradicts to $\zeta$ being a simulation function. So $d\left(T^{n+1} \chi, T^{n} \chi\right)<d\left(T^{n} \chi, T^{n-1} \chi\right)$ holds. This means $\left\{\mathrm{d}\left(\mathrm{T}^{\mathrm{n}} \mathrm{x}, \mathrm{T}^{\mathrm{n}-1} \mathrm{x}\right)\right\}$ is a monotonically decreasing nonnegative sequence.

Let $\lim _{n \rightarrow \infty} d\left(T^{n} x, T^{n-1} \chi\right)=r$. If $r>0$ then by $(\zeta 3)$ and by the contraction condition

$$
\begin{aligned}
0 & \leqslant \limsup _{n \rightarrow \infty} \zeta\left(d\left(T^{n+1} x, T^{n} x\right), M\left(T^{n} x, T^{n-1} x\right)\right) \\
& =\limsup _{n \rightarrow \infty} \zeta\left(d\left(T^{n+1} x, T^{n} x\right), d\left(T^{n} x, T^{n-1} x\right)\right)<0,
\end{aligned}
$$

which is contradiction. Thus $r=0$ and $T$ is asymptotically regular.

Theorem 2.3. Every generalized $\mathrm{Z}$ contraction mapping on a complete $\mathrm{T}_{1}$-quasi metric space has a unique fixed point and every Picard sequence converges to its unique fixed point.

Proof. Let $(X, d)$ be a complete $T_{1}$-quasi metric space and let $T: X \rightarrow X$ be a generalized $Z$ contraction with respect to $\zeta \in Z$. First, let us show that if $T$ has a fixed point, then it is unique.

Suppose that there are two fixed points $z, w \in X$ of the mapping $T$, then $d(z, w)>0$. By $(2.1)$

$$
\zeta(\mathrm{d}((\mathrm{T} z, \mathrm{~T} w), \mathrm{M}(z, w))) \geqslant 0,
$$

where

$$
M(z, w)=\max \left\{d(z, w), d(z, z), d(w, w), \frac{1}{2}(d(z, w)+d(z, w))\right\}=d(z, w),
$$

which contradicts to ( $\zeta 2)$. Thus the fixed point is unique.

Now we are going to prove if $\left\{x_{n}\right\}$ is a Picard sequence generated by $T$, then $\lim _{n \rightarrow \infty} x_{n}=z$ is the unique fixed point of $T$. Let $x_{0} \in X$ be an arbitrary point and $\left\{x_{n}\right\}$ be the Picard sequence, that is, $x_{n+1}=T x_{n}$, for all $n \in \mathbb{N}$. To prove $D=\left\{d\left(x_{n}, x_{m}\right), n, m \in \mathbb{N}\right\}$ is bounded, let $D=D_{1} \cup D_{2} \cup\{0\}$ where $D_{1}=\left\{d\left(x_{n}, x_{m}\right), n>m \in \mathbb{N}\right\}$ and $D_{2}=\left\{d\left(x_{n}, x_{m}\right), n<m \in \mathbb{N}\right\}$.

Suppose $D_{1}$ is not bounded. If there exists some $n \geqslant 0$ and $p \geqslant 1$ such that $x_{n}=x_{n+p}$ then the set $\left\{x_{n}: n \in \mathbb{N}\right\}$ is finite and $D_{1}$ is bounded. Hence assume that for all $n, m \in \mathbb{N}$, if $n \neq m$, then $x_{n} \neq x_{m}$. It is known from Lemma 2.2 that $\mathrm{T}$ is asymptotically regular, accordingly we know that

$$
0<d\left(x_{n+1}, x_{n}\right)<d\left(x_{n}, x_{n-1}\right),
$$




$$
\begin{aligned}
& 0<d\left(x_{n}, x_{n+1}\right)<d\left(x_{n-1}, x_{n}\right), \\
& r=\lim _{n \rightarrow \infty} d\left(x_{n+1}, x_{n}\right)=\lim _{n \rightarrow \infty} d\left(x_{n}, x_{n+1}\right)=0 .
\end{aligned}
$$

Since the limit equals to 0 , there exists an $n_{0} \in \mathbb{N}$ such that $d\left(x_{n+1}, x_{n}\right)<1$ for all $n \geqslant n_{0}$. As $D_{1}$ is not bounded, we can find $n_{1}>n_{0}$ such that $d\left(x_{n_{1}}, x_{n_{0}}\right)>1$. If $n_{1}$ is the smallest natural number greater than $n_{0}$ verifying this property, then we conclude that $d\left(x_{n_{1}}, x_{n_{0}}\right)>1$ and $d\left(x_{p}, x_{n_{0}}\right) \leqslant 1$, for all $p \in$ $\left\{n_{0}, n_{0}+1, \cdots, n_{1}-1\right\}$. Again, since $D_{1}$ is not bounded, there exists $n_{2}>n_{1}$ such that $d\left(x_{n_{2}}, x_{n_{1}}\right)>1$ and $d\left(x_{p}, x_{n_{1}}\right) \leqslant 1$, for all $p \in\left\{n_{1}, n_{1}+1, \cdots, n_{2}-1\right\}$. Repeating this process, we have a partial subsequence $\left\{x_{n_{k}}\right\}$ of $\left\{x_{n}\right\}$ such that for all $k \geqslant 1$

$$
d\left(x_{n_{k+1}}, x_{n_{k}}\right)>1, d\left(x_{p}, x_{n_{k}}\right) \leqslant 1, \quad p \in\left\{n_{k}, n_{k}+1, \cdots, n_{k+1}-1\right\} .
$$

By triangle inequality we have

$$
\begin{aligned}
& 1<d\left(x_{n_{k+1}}, x_{n_{k}}\right) \leqslant d\left(x_{n_{k+1}}, x_{n_{k+1}-1}\right)+d\left(x_{n_{k+1}-1}, x_{n_{k}}\right) \\
& 1<d\left(x_{n_{k+1}}, x_{n_{k}}\right) \leqslant d\left(x_{n_{k+1}}, x_{n_{k+1}-1}\right)+1 .
\end{aligned}
$$

As $k \rightarrow \infty$, by Squeeze Theorem, $\lim _{n \rightarrow \infty} d\left(x_{n_{k+1}}, x_{n_{k}}\right)=1$, similarly we have

$$
\begin{gathered}
\lim _{n \rightarrow \infty} d\left(x_{n_{k+1}-1}, x_{n_{k}-1}\right)=1, \\
M\left(x_{n_{k+1}-1}, x_{n_{k}-1}\right)=\max \left\{d\left(x_{n_{k+1}-1}, x_{n_{k}-1}\right), d\left(x_{n_{k+1}}, x_{n_{k+1}-1}\right), d\left(x_{n_{k}}, x_{n_{k}-1}\right),\right. \\
\left.\frac{1}{2}\left\{d\left(x_{n_{k+1}-1}, x_{n_{k}}\right)+d\left(x_{n_{k+1}}, x_{n_{k}-1}\right)\right\}\right\} .
\end{gathered}
$$

It is obvious that

$$
M\left(x_{n_{k+1}-1}, x_{n_{k}-1}\right) \neq d\left(x_{n_{k+1}}, x_{n_{k+1}-1}\right),
$$

or

$$
M\left(x_{n_{k+1}-1}, x_{n_{k}-1}\right) \neq d\left(x_{n_{k}}, x_{n_{k}-1}\right),
$$

since $\lim _{n \rightarrow \infty} d\left(x_{n+1}, x_{n}\right)=0$. The other cases are given below:

Case 1: If $M\left(x_{n_{k+1}-1}, x_{n_{k}-1}\right)=d\left(x_{n_{k+1}-1}, x_{n_{k}-1}\right)$, since we have $d\left(x_{n_{k+1}-1}, x_{n_{k}-1}\right) \leqslant d\left(x_{n_{k+1}-1}, x_{n_{k}}\right)+$ $d\left(x_{n_{k}}, x_{n_{k}-1}\right)$, where $d\left(x_{n_{k+1}-1}, x_{n_{k}}\right) \leqslant 1$ and $d\left(x_{n_{k}}, x_{n_{k}-1}\right) \rightarrow 0$ for $n \rightarrow \infty$, we conclude that

$$
M\left(x_{n_{k+1}-1}, x_{n_{k}-1}\right) \leqslant 1 .
$$

Case 2: If $M\left(x_{n_{k+1}-1}, x_{n_{k}-1}\right)=\frac{1}{2}\left\{d\left(x_{n_{k+1}-1}, x_{n_{k}}\right)+d\left(x_{n_{k+1}}, x_{n_{k}-1}\right)\right\}$,

$$
\begin{aligned}
M\left(x_{n_{k+1}-1}, x_{n_{k}-1}\right) & \leqslant \frac{1}{2}\left\{1+d\left(x_{n_{k+1}}, x_{n_{k}-1}\right)\right\} \\
& \leqslant \frac{1}{2}\left\{1+d\left(x_{n_{k+1}}, x_{n_{k+1}-1}\right)+d\left(x_{n_{k+1}-1}, x_{n_{k}}\right)+d\left(x_{n_{k}}, x_{n_{k}-1}\right)\right\} .
\end{aligned}
$$

It can be seen that $d\left(x_{n_{k+1}-1}, x_{n_{k}}\right) \leqslant 1$, both $d\left(x_{n_{k+1}-1}, x_{n_{k+1}-1}\right)$ and $d\left(x_{n_{k}}, x_{n_{k}-1}\right) \rightarrow 0$ as $k \rightarrow \infty$ which shows $M\left(x_{n_{k+1}-1}, x_{n_{k}-1}\right) \leqslant 1$ in this case. Therefore,

$$
\lim _{n \rightarrow \infty} d\left(x_{n_{k+1}-1}, x_{n_{k}-1}\right) \leqslant \lim _{k \rightarrow \infty} M\left(x_{n_{k+1}-1}, x_{n_{k}-1}\right) \leqslant 1,
$$

and

$$
1 \leqslant \lim _{k \rightarrow \infty} M\left(x_{n_{k+1}-1}, x_{n_{k}-1}\right) \leqslant 1 .
$$


This means $\lim _{k \rightarrow \infty} M\left(x_{n_{k+1}-1}, x_{n_{k}-1}\right)=1$. From ( $\left.\zeta 3\right)$ and the contraction condition, we have

$$
0 \leqslant \limsup _{k \rightarrow \infty} \zeta\left(d\left(x_{n_{k+1}}, x_{n_{k}}\right), M\left(x_{n_{k+1}-1}, x_{n_{k}-1}\right)\right)<0,
$$

which is a contradiction. This contradiction concludes that $\mathrm{D}_{1}$ is bounded. Similarly, it can be proved that $\mathrm{D}_{2}$ is also bounded. Therefore $\mathrm{D}$ is bounded.

We need to show that $\left\{x_{n}\right\}$ is a left Cauchy sequence. Let $c_{n}=\sup \left\{d\left(x_{i}, x_{j}\right): i \geqslant j \geqslant n\right\}$. We know that $\left\{c_{n}\right\}$ is a monotonically nonincreasing sequence of nonnegative real numbers therefore it is convergent to a nonnegative real number $c=\lim _{n \rightarrow \infty} c_{n}$. We are going to show that $c=0$, assume to the contrary, $c>0$, then by definition of $c_{n}$,

$$
\forall k \in \mathbb{N} \exists m_{k}>n_{k} \geqslant k \quad \text { such that } c_{k}-\frac{1}{k}<d\left(x_{m_{k}}, x_{n_{k}}\right) \leqslant c_{k} \Rightarrow \lim _{k \rightarrow \infty} d\left(x_{m_{k}}, x_{n_{k}}\right)=c .
$$

We know that

$$
\begin{aligned}
d\left(x_{m_{k}}, x_{n_{k}}\right) & \leqslant d\left(x_{m_{k-1}}, x_{n_{k-1}}\right) \\
& \leqslant d\left(x_{m_{k-1}}, x_{m_{k}}\right)+d\left(x_{m_{k}}, x_{n_{k}}\right)+d\left(x_{n_{k}}, x_{n_{k-1}}\right),
\end{aligned}
$$

and

$$
\mathrm{d}\left(\mathrm{x}_{\mathrm{m}_{\mathrm{k}-1}}, x_{\mathrm{m}_{\mathrm{k}}}\right) \rightarrow 0, \mathrm{~d}\left(\mathrm{x}_{\mathrm{n}_{\mathrm{k}}}, x_{\mathrm{n}_{\mathrm{k}-1}}\right) \rightarrow 0,
$$

as $k \rightarrow \infty$. Then by Squeeze Theorem, we have $\lim _{k \rightarrow \infty} d\left(x_{m_{k-1}}, x_{n_{k-1}}\right)=c$ as well.

$$
\begin{gathered}
M\left(x_{m_{k-1}}, x_{n_{k-1}}\right)=\max \left\{d\left(x_{m_{k-1}}, x_{n_{k}-1}\right), d\left(x_{m_{k}}, x_{m_{k-1}}\right), d\left(x_{n_{k}}, x_{n_{k-1}}\right),\right. \\
\left.\frac{1}{2}\left\{d\left(x_{m_{k-1}}, x_{n_{k}}\right)+d\left(x_{m_{k}}, x_{n_{k}-1}\right)\right\}\right\} .
\end{gathered}
$$

We know that $d\left(x_{m_{k}}, x_{m_{k-1}}\right) \rightarrow 0, d\left(x_{n_{k}}, x_{n_{k-1}}\right) \rightarrow 0$ as $k \rightarrow \infty$. Therefore there are two cases:

Case 1: If $M\left(x_{m_{k-1}}, x_{n_{k-1}}\right)=d\left(x_{m_{k-1}}, x_{n_{k}-1}\right)$, then $M\left(x_{m_{k-1}}, x_{n_{k-1}}\right) \rightarrow c$ as $k \rightarrow \infty$.

Case 2: If $M\left(x_{m_{k-1}}, x_{n_{k-1}}\right)=\frac{1}{2}\left\{d\left(x_{m_{k-1}}, x_{n_{k}}\right)+d\left(x_{m_{k}}, x_{n_{k}-1}\right)\right\}$, then we have

$$
M\left(x_{m_{k-1}}, x_{n_{k-1}}\right) \leqslant \frac{1}{2}\left\{d\left(x_{m_{k-1}}, x_{n_{k-1}}\right)+d\left(x_{n_{k-1}}, x_{n_{k}}\right)+d\left(x_{m_{k}}, x_{n_{k}}\right)+d\left(x_{n_{k}}, x_{n_{k-1}}\right)\right\} .
$$

Since $d\left(x_{m_{k-1}}, x_{n_{k-1}}\right) \rightarrow c, d\left(x_{n_{k-1}}, x_{n_{k}}\right) \rightarrow 0, d\left(x_{m_{k}}, x_{n_{k}}\right) \rightarrow c, d\left(x_{n_{k}}, x_{n_{k-1}}\right) \rightarrow 0$ as $k \rightarrow \infty$, we conclude that $M\left(x_{m_{k-1}}, x_{n_{k-1}}\right) \rightarrow c$.

By $(\zeta 3)$ and the contraction condition we have

$$
0 \leqslant \limsup _{k \rightarrow \infty} \zeta\left(d\left(x_{m_{k}}, x_{n_{k}}\right), M\left(x_{m_{k-1}}, x_{n_{k-1}}\right)\right)<0 .
$$

This contradiction proves $c=0$. Therefore $\left\{x_{n}\right\}$ is a left Cauchy sequence. Similarly right Cauchyness can be proved. Since $X$ is complete, there exists $z \in X$ such that $\lim _{n \rightarrow \infty} x_{n}=z$. We are going to show that $z$ is the fixed point of $T$. Suppose $T z \neq z$, then $d(T z, z)>0$, we have

$$
\lim _{n \rightarrow \infty} d\left(T z, T x_{n}\right)=\lim _{n \rightarrow \infty} d\left(T z, x_{n+1}\right)=d(T z, z)>0,
$$

and

$$
\begin{aligned}
M\left(z, x_{n}\right) & =\max \left\{d\left(z, x_{n}\right), d(T z, z), d\left(T x_{n}, x_{n}\right), \frac{1}{2}\left\{d\left(z, T x_{n}\right)+d\left(T z, x_{n}\right)\right\}\right\} \\
& =\max \left\{d\left(z, x_{n}\right), d(T z, z), d\left(x_{n+1}, x_{n}\right), \frac{1}{2}\left\{d\left(z, x_{n+1}\right)+d\left(T z, x_{n}\right)\right\}\right\} .
\end{aligned}
$$

Therefore, $M\left(z, x_{n}\right) \rightarrow d(T z, z)$ as $n \rightarrow \infty$. By contraction condition,

$$
0 \leqslant \zeta\left(\mathrm{d}\left(\mathrm{T} z, \mathrm{~T} x_{\mathrm{n}}\right), \mathrm{M}\left(z, x_{\mathrm{n}}\right)\right) \rightarrow \zeta(\mathrm{d}(\mathrm{T} z, z), \mathrm{d}(\mathrm{T} z, z)) \text { as } \mathrm{n} \rightarrow \infty .
$$

By $(\zeta 2)$, we have $\zeta(d(T z, z), d(T z, z))<0$ which contradicts the contraction condition. That means $\mathrm{T} z=z$ and $z$ is the unique fixed point of $\mathrm{T}$. 
Example 2.4. Let $X=\left[0, \frac{1}{3}\right]$ and

$$
d(x, y)= \begin{cases}y-x, & \text { if } x \leqslant y \\ x, & \text { if } x>y\end{cases}
$$

Define $\mathrm{T}: \mathrm{X} \rightarrow \mathrm{X}$ by $\mathrm{T} x=\frac{x}{2}$ and the simulation function $\zeta:[0, \infty) \times[0, \infty) \rightarrow \mathbb{R}$ by $\zeta(\mathrm{t}, \mathrm{s})=\frac{1}{2} s-\mathrm{t}$.

(i) If $x \leqslant \frac{y}{2}$, then $M(x, y)=\max \left\{y-x, \frac{x}{2}, \frac{y}{2}, \frac{1}{2}\left(y-\frac{x}{2}+\frac{y}{2}-x\right)\right\}=y-x$ and $\zeta(d(T x, T y), M(x, y))=$ $\zeta\left(\left(\frac{y}{2}-\frac{x}{2}\right), y-x\right)=0$.

(ii) If $\frac{y}{2}<x \leqslant y$, then $M(x, y)=\max \left\{y-x, \frac{x}{2}, \frac{y}{2}, \frac{1}{2}\left(y-\frac{x}{2}+x\right)\right\}=\frac{2 y+x}{4}$ and $\zeta(d(T x, T y), M(x, y))=$ $\frac{2 y+x}{8}-\left(\frac{y}{2}-\frac{x}{2}\right)>0$.

(iii) If $y \leqslant \frac{x}{2}$ then $M(x, y)=\max \left\{x, \frac{x}{2}, \frac{y}{2}, \frac{1}{2}\left(\frac{x}{2}+x\right)\right\}=x$ and $\zeta(d(T x, T y), M(x, y))=\frac{x}{2}-\frac{x}{2}=0$.

(iv) If $\frac{x}{2}<y \leqslant x$ then $M(x, y)=\max \left\{x, \frac{x}{2}, \frac{y}{2}, \frac{1}{2}\left(y-\frac{x}{2}+x\right)\right\}=x$ and $\zeta(d(T x, T y), M(x, y))=0$.

$\zeta(d(T x, T y), M(x, y)) \geqslant 0$ holds in any case, therefore $T$ is a generalized $Z$ contraction with respect to $\zeta$ and its obvious unique fixed point is $z=0$.

\section{References}

[1] H. H. Alsulami, E. Karapınar, F. Khojasteh, A. F. Roldán-López-de-Hierro, A proposal to the study of contractions in quasi-metric spaces, Discrete Dyn. Nat. Soc., 2014 (2014), 10 pages. 1

[2] I. Altun, G. Minak, M. Olgun, Classification of completeness of quasi metric space and some new fixed point results, J. Nonlinear Funct. Anal., (Submitted). 1

[3] S. Banach, Sur les opérations dans les ensembles abstraits et leur application aux équations intégrales, Fund. Math., 3 (1922), 133-181. 1

[4] F. E. Browder, W. V. Petryshyn, The solution by iteration of nonlinear functional equations in Banach spaces, Bull. Amer. Math. Soc., 72 (1966), 571-575. 1, 1.10

[5] V. W. Bryant, A remark on a fixed-point theorem for iterated mappings, Amer. Math. Monthly, 75 (1968), 399-400.

[6] J. Caristi, Fixed point theorems for mappings satisfying inwardness conditions, Trans. Amer. Math. Soc., 215 (1976), 241-251.

[7] L. B. Ćirić, On a common fixed point theorem of a Greguš type, Publ. Inst. Math. (Beograd) (N.S.), 49 (1991), $174-178$. 1

[8] S. Cobzaş, Completeness in quasi-metric spaces and Ekeland Variational Principle, Topology Appl., 158 (2011), 10731084. 1

[9] K. Deimling, Multivalued differential equations, De Gruyter Series in Nonlinear Analysis and Applications, Walter de Gruyter \& Co., Berlin, (1992). 1

[10] M. Edelstein, A theorem on fixed points under isometries, Amer. Math. Monthly, 70 (1963), 298-300. 1

[11] T. L. Hicks, Fixed point theorems for quasi-metric spaces, Math. Japon., 33 (1988), 231-236. 1

[12] O. Kada, T. Suzuki, W. Takahashi, Nonconvex minimization theorems and fixed point theorems in complete metric spaces, Math. Japon., 44 (1996), 381-391. 1

[13] R. Kannan, Some results on fixed points, II, Amer. Math. Monthly, 76 (1969), 405-408. 1

[14] F. Khojasteh, S. Shukla, S. Radenović, A new approach to the study of fixed point theory for simulation functions, Filomat, 29 (2015), 1189-1194. 1

[15] M. Olgun, Ö. Biçer, T. Alyildiz, A new aspect to Picard operators with simulation functions, Turkish J. Math., 40 (2016), 832-837. 1

[16] I. L. Reilly, P. V. Subrahmanyam, M. K. Vamanamurthy, Cauchy sequences in quasipseudometric spaces, Monatsh. Math., 93 (1982), 127-140. 1, 1

[17] B. E. Rhoades, A comparison of various definitions of contractive mappings, Trans. Amer. Math. Soc., 226 (1977), $257-$ 290. 1, 2

[18] A. F. Roldan Lopez de Hierro, B. Samet, $\varphi$-admissibility results via extended simulation functions, J. Fixed Point Theory Appl., 2016 (2016), 19 pages. 1

[19] J. L. Sieber, W. J. Pervin, Completeness in quasi-uniform spaces, Math. Ann., 158 (1965), 79-81. 1

[20] P. V. Subrahmanyam, Completeness and fixed-points, Monatsh. Math., 80 (1975), 325-330. 1

[21] W. A. Wilson, On quasi-metric spaces, Amer. J. Math., 53 (1931), 675-684. 1 